\title{
22X mask cleaning effects on EUV lithography process and lifetime
}

\author{
Simi A. George*a, Robert J. Chen ${ }^{\mathrm{b}}$, Lorie-Mae Baclea-an ${ }^{\mathrm{a}}$ and Patrick P. Naulleau ${ }^{\mathrm{a}}$ \\ ${ }^{a}$ Center for X-ray Optics, Lawrence Berkeley National Laboratory, Berkeley, CA, 94720 \\ ${ }^{\mathrm{b}}$ Intel Corporation, Santa Clara, CA 95052 \\ *Now at SCHOTT North America, Duryea, PA 18642 \\ E-mail: simi.george@us.schott.com, robert.j.chen@intel.com,pnaulleau@lbl.gov
}

\begin{abstract}
For this paper, we evaluated the impact of repetitive cleans on a photomask that was fabricated and patterned for extreme ultraviolet lithography exposure. The lithographic performance of the cleaned mask, in terms of process window and line edge roughness, was monitored with the SEMATECH Berkeley micro-exposure tool (MET). Each process measurement of the cleaned mask was compared to a reference mask with the same mask architecture. Both masks were imaged on the same day in order to eliminate any process-related measurement uncertainties. The cleaned mask was periodically monitored with atomic force microscopy (AFM) measurements and pattern widths were monitored using scanning electron microscopy (SEM). In addition, reflectivity changes were also tracked with the aid of witness plate measurements. At the conclusion of this study, the mask under evaluation was cleaned 22 times; with none of the evaluation techniques showing any significant degradation in performance.
\end{abstract}

Keywords: EUVL, mask, chemical cleaning, process performance

\section{INTRODUCTION}

The reflective, multilayer based, extreme ultraviolet lithography (EUVL) mask architectures are highly susceptible to surface oxidation and contamination. As a result, EUV masks will require periodic cleans processing to mitigate defectivity--as many as twenty times or more to meet high volume manufacturing (HVM) lifetime goals. Mask surface damage and the increased line edge roughness (LER) that may result from these repetitive cleanings remain a concern; thus.mask cleaning processes to be implemented for HVM must have negligible impact on lithographic performance after the many clean cycles that may be needed.

We have previously reported a lithographic performance comparison of a carbon-contaminated mask that was cleaned to a new generation uncontaminated mask [1]. The results of that study showed that the performance was not impacted by the cleaning process and that the cleaning did effectively remove carbon deposits from the mask pattern sidewalls. Another unpublished study involved a mask which was imaged before and after cleaning. The resist LER was observed to be significantly increased after the mask underwent intentionally aggressive multiple cleans processing.

In the studies completed for this paper, we evaluate a wet chemical cleans process designed for EUV mask contamination removal. The target here was to determine the number of times this recipe could be used to clean a standard EUV mask before the multilayer performance began to degrade. Any multilayer or absorber pattern degradation will adversely affect the image quality and photon throughput from the mask. The initial results from these studies were reported recently [2], in which we found that after a total of eight cleans of the mask, statistically quantifiable changes were not observed in any of the completed analysis. 


\section{MATERIALS AND MEASUREMENTS}

Two masks are used for the duration of the studies with one of the masks undergoing repeated cleaning and the other one being kept as a reference. Lithographic performance, in terms of process window analysis and line edge roughness, was monitored after every 2-4 cleans and compared to the reference mask performance. Mask surface changes are monitored by atomic force microscopy (AFM) and scanning electron microscopy (SEM). Reflectivity changes are monitored by xray reflectometry and by witness plate measurements.

\subsection{Mask}

At the beginning of this study, two masks with identical multilayer, capping layer, absorber, and anti-reflection coating architectures are patterned for EUV lithography micro-exposure studies. The capping layer used is Ruthenium (Ru) at $2.5 \mathrm{~nm}$ thickness. Any carbon contamination on the surface of either mask should be minimal, since they have not been used prior to this set of imaging studies. An industry-developed cleaning process is used repeatedly on one of the masks, while the other mask is kept as is.

\subsection{Cleaning}

Standard acid-based wet cleaning chemistries were used to clean the surface of the mask. The process includes two main steps: organic removal by a mixture of sulfuric acid and hydrogen peroxide (SPM) followed by a particle clean by SC1 with megasonics.

\subsection{Process Studies}

The imaging was performed on the SEMATECH Berkeley MET installed at the Advanced Light Source synchrotron facility at Lawrence Berkeley National Laboratory. Details on the MET can be found in references[3-4]. In the lithographic results presented here, all imaging is completed using an annular illumination with an inner sigma of 0.35 and an outer sigma of 0.55 . The illumination angle of incidence is 4 degrees parallel to the mask patterns.

Throughout the imaging studies, we used the same area of the mask. The resist used for the entire study was the BBR08A, which is one of the baseline resists for the MET. This resist was spin-coated onto a wafer primed with the Hexamethyldisilazane (HMDS) adhesion promoter to produce a nominal film thickness of $60 \mathrm{~nm}$. The patterns on resist were recorded using a top-down SEM (Hitachi FE-SEM S-4800) and analyzed offline with the software package SuMMIT[5]. Each data set consisted of an $11 \times 11$ grid of focus and exposure dose step called the focus exposure matrix (FEM). The focus step size used in the FEM was $50 \mathrm{~nm}$ and the dose steps were 5\% exponential.

Process data for two sets of vertical, 1:1 line and space patterns were collected for each mask, one set at the critical dimension (CD) of $40 \mathrm{~nm}$ and the other at a $\mathrm{CD}$ of $36 \mathrm{~nm}$. When the cleaned mask was imaged (referred to as the test mask), the reference mask was also imaged enabling us to monitor any system and process related effects over the long time span of this study.

\subsection{Mask Multilayer and Surface Characterizations}

In order to monitor damage to the Ru capping layer surface, three dimensional mask surface profiles were obtained using a Digital Instruments atomic force microscope at periodic intervals during the cleans. This AFM is capable of measuring sub-angstrom level root mean squared (rms) roughness of a surface, with a nominal tip radius of $8 \mathrm{~nm}$ and a maximum of $12 \mathrm{~nm}$. The imaging is completed in tapping mode with minimal damage to the Ru surface. Each AFM image is $256 \times 256$ pixels, covering an area of $2 \mu \mathrm{m} \times 2 \mu \mathrm{m}$ giving a lateral resolution of approximately $7.8 \mathrm{~nm}$ per pixel. Post processing is used to de-convolve the AFM tip radius from the raw data.

Mask pattern widths were measured on the test mask after every cleans processing cycle with a Hitachi SEM. To track EUV reflectivity changes, a witness plate was fabricated with the same architecture as the test mask. This witness mask was subjected to the same cleaning process and its EUV reflectivity measured at periodic intervals with an EUV Technology reflectometer. 


\section{RESULTS}

\subsection{Pattern performance after 22 cleans}

All of the process metrics extracted from the collected images are compiled in table 1, including exposure latitude percent (EL), depth of focus (DOF), and LER. The DOF is determined based on an elliptical fit to the $+/-10 \%$ CDchange process window. For determining the DOF, the ellipse EL is set to have $10 \%$ CD variation.

Table 1: Process comparison of all relevant parameters for 1:140nm and 36nm lines and spaces patterns

\begin{tabular}{|c|c|c|c|c|c|c|c|c|c|c|c|c|}
\hline & \multicolumn{4}{|c|}{ 40nm CD, 1:1 lines } & \multicolumn{5}{c|}{ 36nm CD, 1:1 lines } \\
\hline \multirow{2}{*}{ \# of Cleans } & \multicolumn{3}{|c|}{ Cleaned Mask } & \multicolumn{3}{c|}{ Reference Mask } & \multicolumn{3}{c|}{ Cleaned Mask } & \multicolumn{3}{c|}{ Reference Mask } \\
\cline { 2 - 14 } & EL & DOF & LER & EL & DOF & LER & EL & DOF & LER & EL & DOF & LER \\
\hline 2 & 18.8 & 319.7 & 4.03 & 16.6 & 292.9 & 4.21 & 16.5 & 306.3 & 4.13 & 16.7 & 303.3 & 4.32 \\
\hline 4 & 16.3 & 330.6 & 3.96 & 15.4 & 335.6 & 3.98 & 16.1 & 294.4 & 3.99 & 11.9 & 185.1 & 4.14 \\
\hline 6 & 16.0 & 245.3 & 4.24 & 13.3 & 200.7 & 4.02 & 14.8 & 239.4 & 4.15 & 13.4 & 212.3 & 4.25 \\
\hline 8 & 15.5 & 287.5 & 4.25 & 14.7 & 155.2 & 4.19 & 14.0 & 308.5 & 4.01 & 12.7 & 181.4 & 4.48 \\
\hline 14 & 25.4 & 302.0 & 3.31 & 22.0 & 256.0 & 3.21 & 22.3 & 320.0 & 3.52 & 19.6 & 304.0 & 3.40 \\
\hline 16 & 24.3 & 247.6 & 3.42 & 21.9 & 226.4 & 3.36 & 21.0 & 261.7 & 3.82 & 20.3 & 253.8 & 3.60 \\
\hline 18 & 20.6 & 260.0 & 3.45 & 19.6 & 180.1 & 3.52 & 20.2 & 260.0 & 3.74 & 16.4 & 193.3 & 3.83 \\
\hline 22 & 22.8 & 300.0 & 3.47 & 21.3 & 334.0 & 3.19 & 19.1 & 252.0 & 3.65 & 18.8 & 271.0 & 3.21 \\
\hline
\end{tabular}

EL is in \%, DOF and LER are given in nm units.

Both exposure latitude and DOF data for both pattern widths and all cases show less than 5\% variation. This is well within the process uncertainty, which is expected to be anywhere from 5 to $10 \%$ for normal wafer to wafer MET operation conditions. The EL trend throughout span of the study is plotted in figure 1. It is seen that the reference mask is slightly worse than the cleaned mask and this stays nearly the same towards the end of the study.
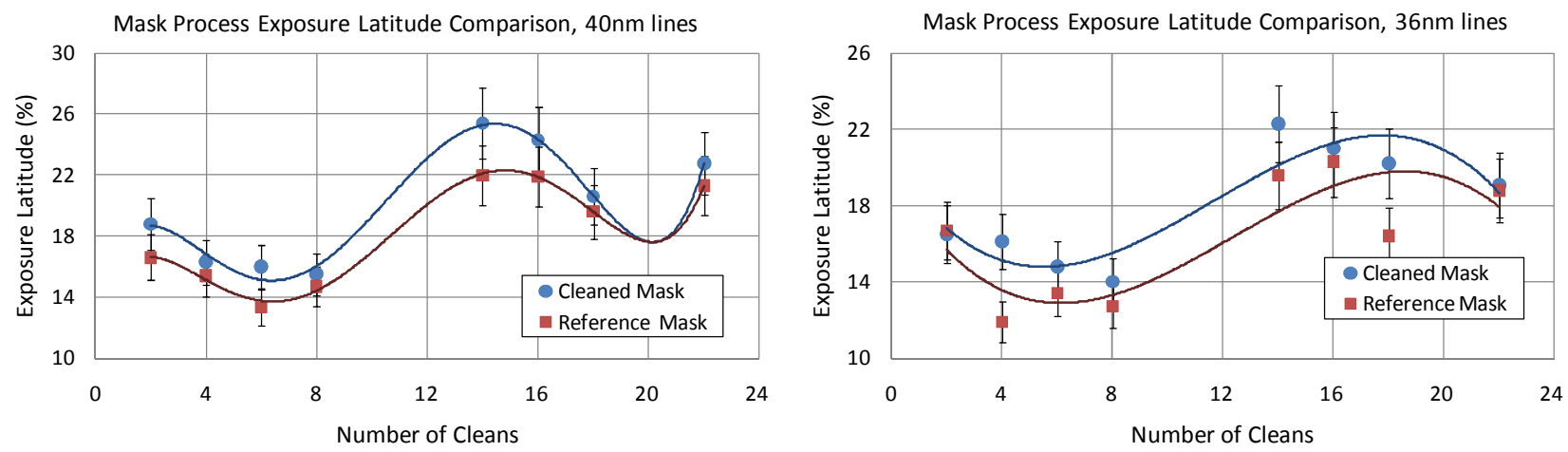

Figure 1, Exposure latitude comparison throughout the mask clean studies for both the cleaned mask and the reference mask. Any system level changes and resist level changes are accounted for by comparing the two masks. Changes observed in the data are within the statistical limit of normal wafer to wafer patterning.

The iso-focal feature widths for both sets of patterns are observed to be nearly identical throughout the study, indicating that there is no damage to the mask patterns. The data is tabulated in table 2 for both line sets evaluated. No evidence of CD change, relative to the uncertainty, is found. That is true both in an absolute sense and relative to the reference mask. The uncertainty in the data is found to be near $2.0 \mathrm{~nm}$. 
Table 2: Iso-focal LER matched and averaged CD comparison for all data for the 40nm and 36nm line widths

\begin{tabular}{|c|c|c|c|c|}
\hline $\begin{array}{c}\text { 40nm } \\
\text { lines }\end{array}$ & \multicolumn{2}{|c|}{$\begin{array}{c}\text { Cleaned } \\
\text { Mask }\end{array}$} & \multicolumn{2}{c|}{$\begin{array}{c}\text { Reference } \\
\text { Mask }\end{array}$} \\
\hline Cleans & $\begin{array}{c}\text { CD_ave } \\
\text { (nm) }\end{array}$ & $\begin{array}{c}\text { E_iso } \\
(\mathbf{m J})\end{array}$ & CD_ave & E_mJ \\
\hline 2 & 38.2 & 15.24 & 39.0 & 14.38 \\
\hline 4 & 37.5 & 15.23 & 39.8 & 14.16 \\
\hline 6 & 39.9 & 14.88 & 38.0 & 13.94 \\
\hline 8 & 39.0 & 15.24 & 40.2 & 14.17 \\
\hline 14 & 38.2 & 18.14 & 39.3 & 19.81 \\
\hline 16 & 37.2 & 16.80 & 39.8 & 16.20 \\
\hline 18 & 39.4 & 16.00 & 39.8 & 17.18 \\
\hline 22 & 38.9 & 16.54 & 39.6 & 15.38 \\
\hline
\end{tabular}

\begin{tabular}{|c|c|c|c|c|}
\hline $\begin{array}{c}\text { 36nm } \\
\text { lines }\end{array}$ & \multicolumn{2}{|c|}{$\begin{array}{c}\text { Cleaned } \\
\text { Mask }\end{array}$} & \multicolumn{2}{c|}{$\begin{array}{c}\text { Reference } \\
\text { Mask }\end{array}$} \\
\hline Cleans & $\begin{array}{c}\text { CD_ave } \\
\text { (nm) }\end{array}$ & $\begin{array}{c}\text { E_iso } \\
(\mathbf{m J})\end{array}$ & CD_ave & E_mJ \\
\hline 2 & 34.4 & 15.24 & 35.2 & 15.10 \\
\hline 4 & 34.5 & 15.23 & 34.1 & 13.81 \\
\hline 6 & 35.2 & 15.63 & 34.1 & 14.29 \\
\hline 8 & 36.1 & 15.24 & 35.4 & 14.51 \\
\hline 14 & 35.9 & 18.14 & 34.8 & 19.81 \\
\hline 16 & 34.7 & 16.80 & 34.4 & 16.80 \\
\hline 18 & 34.6 & 16.80 & 34.6 & 17.60 \\
\hline 22 & 34.6 & 16.54 & 35.3 & 15.75 \\
\hline
\end{tabular}

The averaged, $3 \sigma$ LER can also be found in table 1, where it is seen that the characterized variations in all cases are well below the measurement and calculation uncertainty of $\pm 0.40 \mathrm{~nm}$. The LER is plotted in figure 2, where the data shows similar trends as the exposure latitude, except in this case, the cleaned mask is shown to have slightly worse LER at the smaller line width, though it is still within the MET measurement uncertainty range.
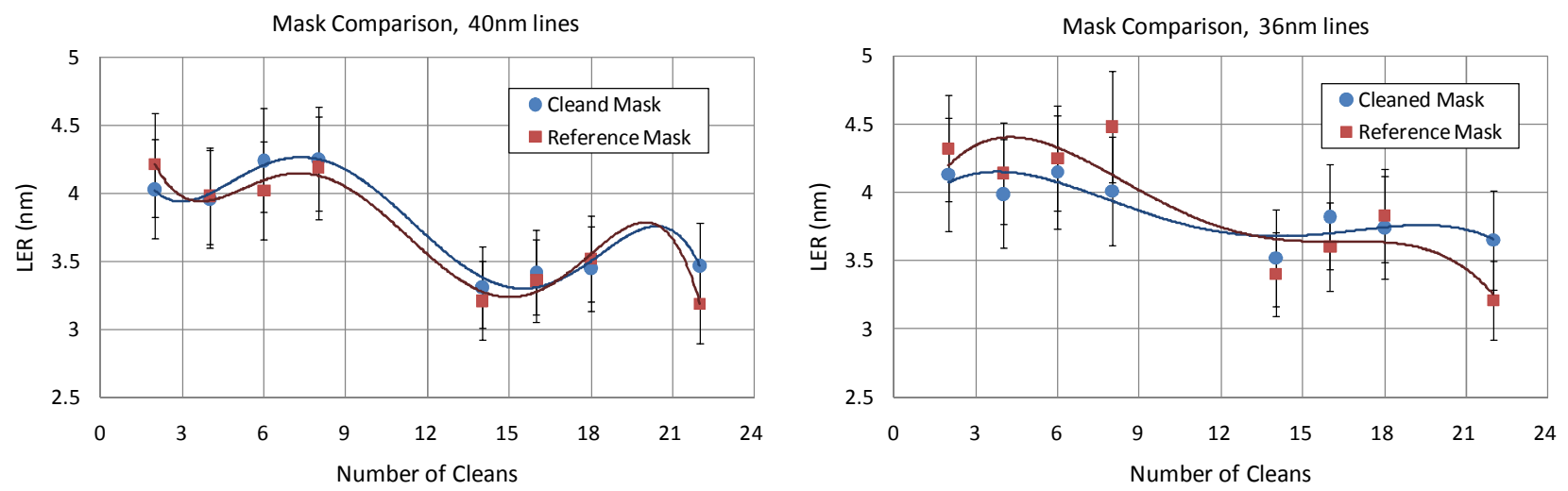

Figure 2, LER trends throughout the mask clean studies for both the cleaned mask and the reference mask.

\subsection{Mask surface after 22 cleans}

The surface topographies recorded with the AFM at fixed intervals of the cleaning cycles were post-processed and analyzed to assess for any detectable capping layer damage. The mask blanks are qualified at the mid-spatial frequency range (MSFR) and these values do not show any notable variations. All tabulated values for roughness are given in table 3 (data after 22 cleans was still in progress as of this writing). EUV reflectivity measurements carried out on the witness mask show that after 23 cleans (figure 3), reflectivity remains unchanged. 
Table 3: Roughness magnitudes extracted from the AFM images of the cleaned mask at set intervals.

\begin{tabular}{|c|c|c|c|c|c|c|c|c|}
\hline & \multicolumn{9}{|c|}{ AFM rms roughness (nm) } \\
\hline \# of Cleans & Initial & 2 & 4 & 8 & 10 & 12 & 14 & 18 \\
\hline $\begin{array}{c}\text { Mid-spatial } \\
\text { frequency }\end{array}$ & 0.069 & 0.069 & 0.079 & 0.062 & 0.067 & 0.064 & 0.066 & 0.064 \\
\hline
\end{tabular}

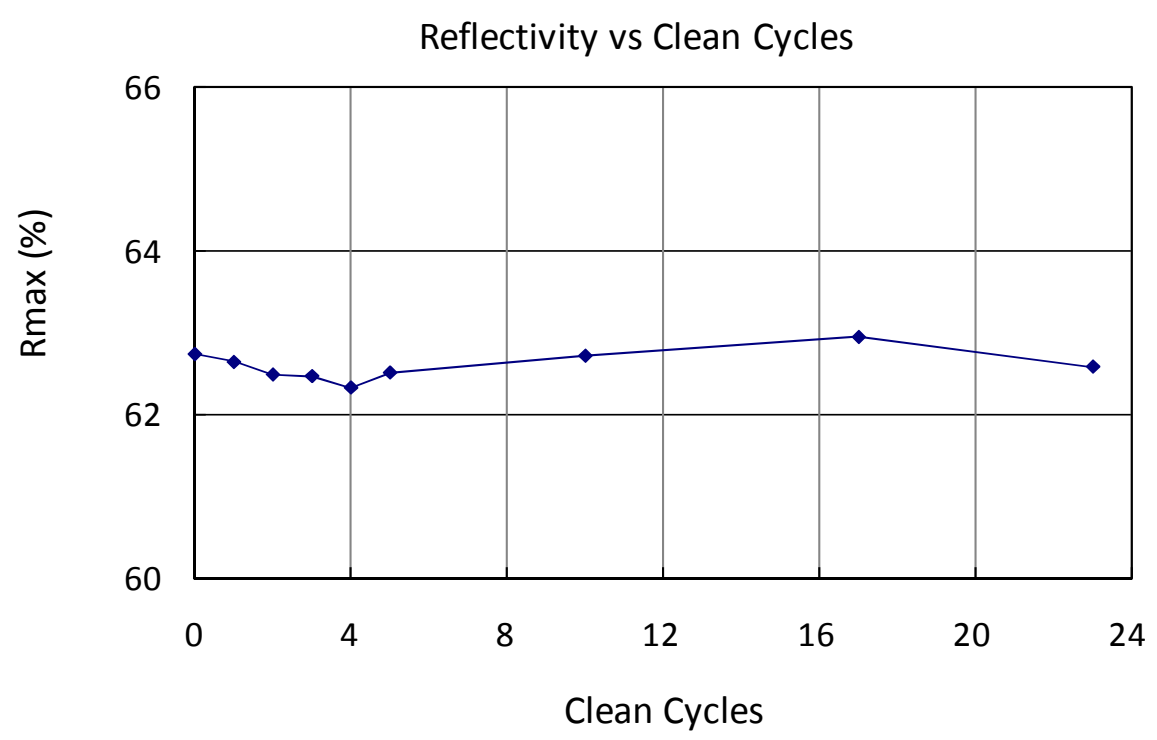

Figure 3, Reflectivity vs Cleans for a total of 23 cleans on the witness plate. The data shows negligible impact on reflectivity.

CD-SEM images of the mask were collected for the 40nm and $36 \mathrm{~nm}$ equivalent lines and spaces on the mask and these were analyzed offline with SUMMIT. Shown in figure 4 are images obtained before the start of cleaning and after the mask was cleaned 22 times. Both $36 \mathrm{~nm}$ and 40nm equivalent lines show little change after 22 cleans, well within the $5 \%$ error expected from SEM measurement variation.

\section{SUMMARY}

The data presented shows minimal degradation in lithographic performance for an EUV mask after 22 cleans with acidbased chemistry. EL comparison between the cleaned mask and the reference mask does not show any change greater in magnitude than were seen at the start of the study when both masks were pristine. The variations in LER are shown to be less than the process uncertainty determined for the resist at $\pm 0.4 \mathrm{~nm}$. Iso-focal CD for patterns evaluated remains the same. Furthermore, surface analysis by AFM did not show any changes in the mid-spatial frequency roughness. Finally, no decrease in EUV reflectivity was seen on the witness mask after 23 cleans. We conclude that the cleans processing completed to this point have not damaged mask multilayer or absorber structures. 


\section{0nm patterns in resist}

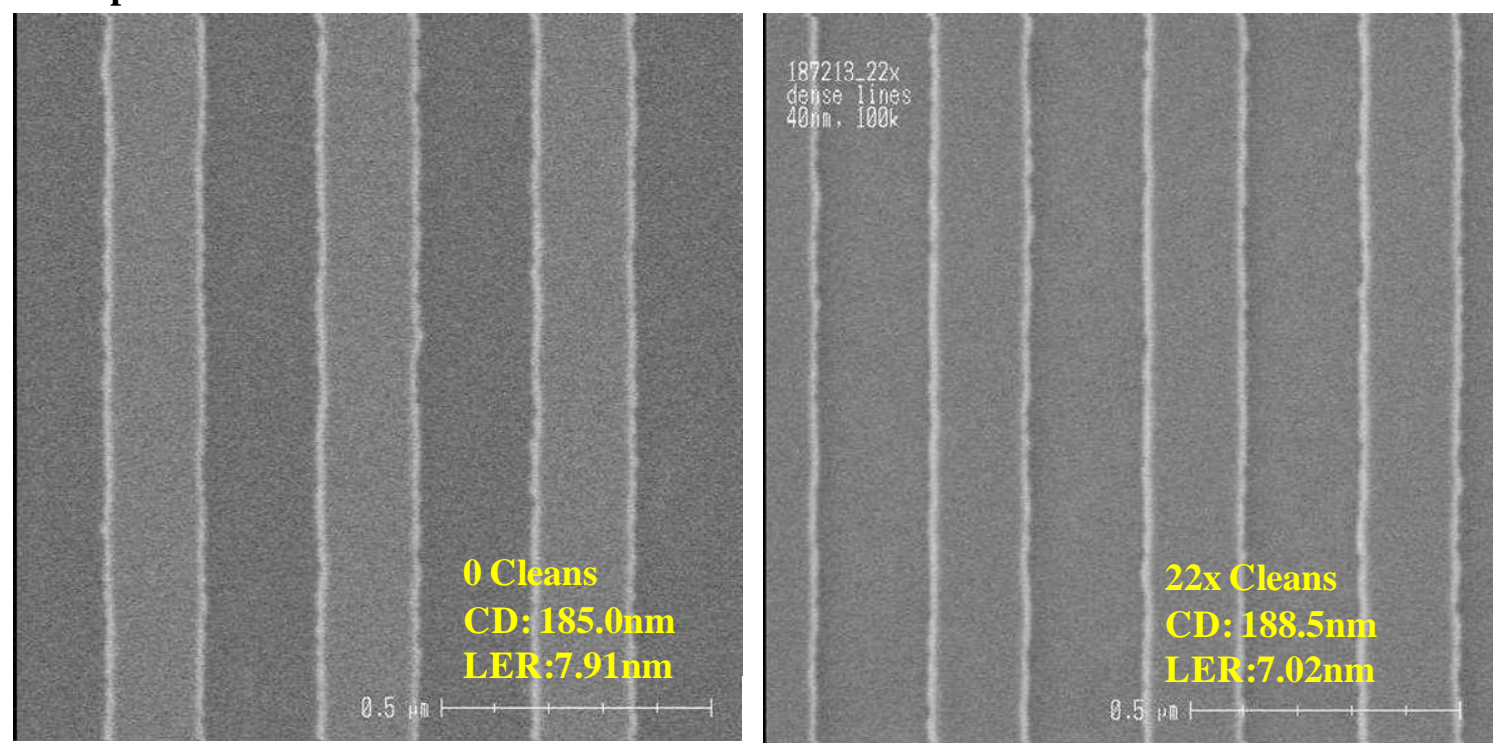

\section{6nm patterns in resist}
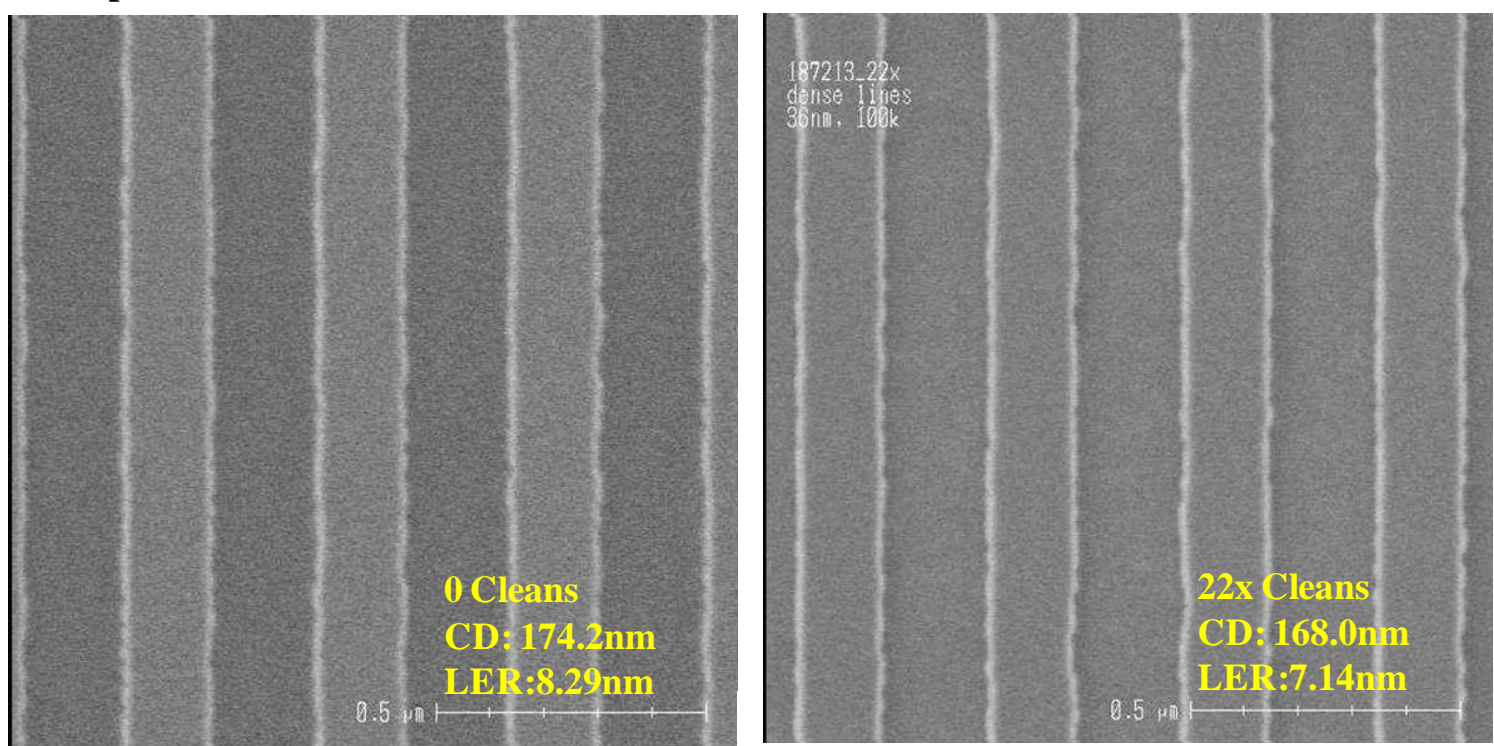

Figure 4: CDSEM images collected before the start of cleans and after the mask was cleaned 22 times. For the 40nm lines, the averaged CD and LER do not show differences beyond measurement related error. The $36 \mathrm{~nm}$ case does show a decrease in CD, but will not be detectable in pattern studies, after MET demagnification and the resist imaging errors are taken into account,

\section{Acknowledgments}

We wish to acknowledge the expert support provided by Paul Denham, Gideon Jones, Brian Hoef, and Christopher N. Anderson of the Center for X-Ray Optics at Lawrence Berkeley National Laboratory with the exposure tool. The entire CXRO engineering team builds and maintains the EUV exposure tool. We acknowledge SEMATECH for the support of the SEMATECH Berkeley MET and in particular the programmatic support from Warren Montgomery, Bryan Rice, and Stefan Wurm. This work was supported in part by SEMATECH and carried out at Lawrence Berkeley National Laboratory's Advanced Light Source, which is supported by the DOE, Office of Science and the Basic Energy Sciences under Contract No. DE-AC02-05CH11231. 


\section{REFERENCES}

[1] Simi George, Patrick Naulleau, Uzodinma Okoroanyanwu, Kornelia Dittmar, Christian Holfeld, and Andrea Wüest, J. Vac. Sci. Technol. B 28, 841 (2010)

[2] Simi A. George, Lorie Mae Baclea-an, Patrick P. Naulleau, Robert J. Chen, and Ted Liang, J. Vac. Sci. Technol. B 28, C6E31 (2010)

[3] P. P. Naulleau, C. N. Anderson, J. Chiu, K. Dean, P. Denham, K. A. Goldberg, B. Hoef, S. Huh, G. Jones, B. M. LaFontaine, A. Ma, D. Niakoula, J. Park, and T. Wallow, Proc. SPIE 6921, 69213N (2008).

[4] P. P. Naulleau, K. A. Goldberg, E. Anderson, J. P. Cain, P. Denham, K. Jackson, A.-S. Morlens, S. Rekawa, and F. Salmassi, J. Vac. Sci. Technol. B 22, 2962 (2004).

[5] SuMMIT Software Division of EUV Technology, Summit Litho Image Analysis Software, 2009. http://www.euvl.com/summit/ 


\section{DISCLAIMER}

This document was prepared as an account of work sponsored by the United States Government. While this document is believed to contain correct information, neither the United States Government nor any agency thereof, nor The Regents of the University of California, nor any of their employees, makes any warranty, express or implied, or assumes any legal responsibility for the accuracy, completeness, or usefulness of any information, apparatus, product, or process disclosed, or represents that its use would not infringe privately owned rights. Reference herein to any specific commercial product, process, or service by its trade name, trademark, manufacturer, or otherwise, does not necessarily constitute or imply its endorsement, recommendation, or favoring by the United States Government or any agency thereof, or The Regents of the University of California. The views and opinions of authors expressed herein do not necessarily state or reflect those of the United States Government or any agency thereof or The Regents of the University of California. 